\title{
Considérations sur la poésie d'Aimé Césaire
}

Lamarana DIALLO

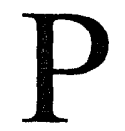

longé dans un univers orphelin et hostile, condamné à périr sous le joug de la servitude et soumis à une existence infernale, le monde noir attendait l'avènement d'un prophète laïc. Déjà il s'impatientait, tant profonde avait été son amertume, grande sa douleur séculaire et angoissante son aliénation. Ainsi, il fallait que des entrailles d'une Martinique en pleurs sortît "un grand poète noir" pour exprimer les frustrations et les rancours d'une race crucifiée par la colonisation et la traite négrière. Et Aimé Césaire, le poète de la souffrance, vint. "Et c'est un Noir qui manie la langue française comme il n'est pas aujourd'hui un Blanc pour la manier." Et c'est un Nègre inconsolé qui apparaît comme le promoteur d'une poésie prométhéenne, d'une poésie qui vise à briser les chaînes du Noir, à lui rendre sa dignité perdue et à lui redonner foi dans l'homme. Et c'est un poète de l'excès, de la démesure et des recherches interdites qui entend revisiter le passé douloureux des Noirs.

Poésie prophétique et évangélique, la poésie d'Aimé Césaire l'est dans l'exacte mesure où elle transmue le poète lyrique et intimiste en un porte-parole de la collectivité noire. Visionnaire, il examine le présent à la lumière d'un passé douloureux en vue d'annoncer des lendemains qui chantent dans une cité future où la béatitude le dispute à la servitude. Cette poésie prophétique semble tirer sal légitimité d'une double fidélité: celle à lia "parole transcendante" et celle "au peuple auquel [le poète] a 
charge de transmettre"'2 ce message. Du reste, il n'en saurait être autrement, d'autant que sa poésie s'affirme comme la mise en évidence d'un thème doloriste, celui d'une race déshumanisée. Dès lors, on conçoit qu'il ait voulu être la "bouche des malheurs qui n'ont point de bouche."3

Bien loin d'être une poésie ornementale, la poésie césairienne semble d'autant plus fonctionnelle qu'elle ne procède aucunement d'une gratuité esthétique. Au vrai, c'est à manifester emphatiquement l'âme noire qu'elle s'emploie. En se faisant l'écho sonore de la conscience nègre, de ses souffrances et de ses haines, elle transcende les exigences de l'art-pourl'art pour exprimer les réalités sociologiques du monde noir:

Pour Césaire, la poésie n'a jamais été séparée de la vie. Il a toujours su qu' elle fournissait à ceux qui ressentent dans leur chair la morsure des ans et la misère du monde, un cadre idéal pour exprimer leur colère et leur désespoir, leurs amours et leurs espérances. ${ }^{4}$

Il est une passion aussi furieuse que dévorante qui parcourt la poésie césairienne. Faisant siennes les aspirations révolutionnaires du Nègre opprimé, cette "poésie d"agriculteurs"s non seulement verse dans une revendication superbe de la "non-technicité," tenants de la culture blanche et de sa raison raisonnante. Sous ce rapport, il est significatif que le poète se soit littéralement gaussé de la prétendue supériorité de l'Occident et qu'il ait éprouvé de la compassion à l'égard d'une Europe malade:

Ecoutez le monde blanc

Horriblement las de son effort immense

Ses articulations rebelles craquer

Sous les étoiles dures

Ses raideurs d'acier bleu

Transperçant la chair mystique

Ecoute ses victoires proditoires trompeter ses défaites

Ecoute aux alibis grandioses son piètre trébuchement

Pitié pour nos vainqueurs omniscients et naîfs!?

Est fortement engagée cette poésie qui fait le procès de l'Europe orgueilleuse. Mais qu'est-ce donc que la poésie? Dans cette perspective, il semble qu'il faille s'interroger sur l'être de la poésie césairienne; il sied de donner à ce dernier l'opportunité de mettre en lumière sa conception de la parole poétique. A ce sujet, il est symptomatique qu'il ait regardé la poésie comme une "démarche qui, par le mot, l'image, le mythe, l'amour et l'humour [l']installe au cœur vivant de [lui]-même et du monde." L'entreprise de dénomination à laquelle fait allusion Césaire vise à prendre 
possession du monde tant par la baguette magique des mots que par les "armes miraculeuses." C'est à faire siennes les puissances des choses que Césaire s'emploie lorsqu'il les nomme. D'autre part, l'imagination du poète demeure orientée vers l'astre du jour autour duquel gravitent nombre d'images symbolisant une source de "lumière, de vie."10 Le recours à la mythologie africaine donne au pape de la négritude la mission de faire revivre le passé du peuple nègre. Toutefois, le regard nostalgique qu'il jette sur l'histoire de sa race reste des plus critiques. Il nie vigoureusement que son peuple ait des origines aussi mythiques que sublimes:

Non, nous n'avons jamais été amazones du roi du Dahomey, ni princes

De Ghana avec huit cents chameaux, ni docteurs à

Tombouctou Askia le Grand étant roi,

Ni architectes de Djenné, ni Madhi. Nous ne sentons pas sous

L'aisselle la démangeaison de ceux qui

Tinrent jadis la lance. Et puisque j'ai

Juré de ne rien celer de notre histoire

Je veux avouer que nous fûmes de tout

Temps d'assez piètres laveurs de vaisselle,

Des cireurs de chaussures sans envergures

Ni guerriers

D'assez consciencieux sorciers et le seul

Indiscutable record que nous ayons

Battu est celui d'endurance à la chicotte."

Cette peinture sans complaisance du passé des Noirs met en exergue une mémoire collective empreinte de blessures tant morales que physiques. Que la poésie de Césaire soit révoltée et douloureuse ne doit plus étonner personne. Toujours est-il que "l'amour tyrannique" qu'il nourrit à l'égard de "ceux qui n'ont inventé ni la poudre ni la boussole"12 transmue la poésie de Césaire en une poésie essentiellement raciale. Ni satirique ni imprécatoire, cette poésie apparaît comme une prise de conscience de la situation abjecte dans laquelle plongent les Noirs. Elle n'est rien de moins qu'un chant de souffrances destiné à ceux qui croupissent dans la misère la plus noire. La poésie césairienne sera "orphique" ou ne sera pas dans l'exacte mesure où le recouvrement de sa dignité insultée demeure fonction de cette descente du Nègre en soi-même:

Ainsi, par un bonheur poétique exceptionnel, c'est en $s^{\prime}$ abandonnant aux transes, en se roulant par terre comme un possédé en prise à soi-même, en chantant ses colères, ses regrets ou ses détestations, en exhibant ses plaies, sa vie déchirée 
entre la "civilisation" et le vieux fond noir; bref en se montrant

le plus lyrique, que le poète noir atteint le plus sûrement à la grande poésie collective. ${ }^{13}$

On comprend qu'il ait usé de l'humour dans l'intention de se gausser des "agresseurs de la race nègre et de secouer les agressés." ${ }^{14}$ Les poèmes de Césaire procèdent d'une veine "militante"15; ils fonctionnent comme une "arme de combat" et s'acharnent à dénoncer avec la dernière énergie les maux aussi multiformes qu'injustifiés dont souffrent les Noirs depuis les temps immémoriaux. Pendant les siècles de plomb de l'esclavage, le Noir a bu la coupe de l'amertume jusqu'à la lie. Le Rebelle qui passe pour être le porte-parole du poète révolté se remémore jusque dans le détail les scènes terrifiantes du passé; les terribles images qui sourdent de ces évocations semblent friser la déraison, tant ignobles sont les actes des tortionnaires blancs:

Et l'on nous vendait comme des bêtes

Et l'on nous comptait les dents...

Et l'on nous tâtait les bourses et

L'on examinait le cati ou décati

De notre peau et l'on nous palpait

Et pesait et soupesait et l'on passait

A notre cou de bête domptée le collier,

De la servitude et du sobriquet. ${ }^{16}$

L'usage de l'accumulation suggère la douleur plurielle à laquelle le Noir est en proie. Du point de vue de la création poétique, l'oppression sans nom subie par le peuple noir au cours de l'esclavage semble positive en tant qu'elle débouche sur une poésie révolutionnaire.

Il est heureux que le thème de l'accusation sous-tende cette production poétique qui a son origine dans "l'existence effective de la douleur éprouvée par le monde noir et de l'humiliation qu'il a subie." ${ }^{17}$ L'époque esclavagiste dépeinte par Césaire apparaît comme le commencement de l'enfer. On conçoit que sa poésie qui semble imprégnée de conscience raciale et qui respire une violence rare, ait cherché à flageller les auteurs de ce fléau inique. En pourfendant ce crime contre l'humanité, Césaire, ce poète de la colère, instruit le procès de l'occident. Au nom de son peuple qui est considéré comme une non-valeur, le porte-parole des "damnés de la terre" accuse le Nord d'avoir violé le sol nègre, d'avoir profané les religions traditionnelles, d'avoir humilié la race noire en la chosifiant, d'avoir assassiné les immenses possibilités artistiques et humaines qui dormaient en lui. 
A cette poésie pamphlétaire et iconoclaste qui se recommande par un lyrisme collectif, succède une forme poétique sibylline et décriée. Dans cette optique, il est éloquent que tous les exégètes de la poésie césairienne se soient accordés pour en souligner le caractère hermétique. L'impressionnante étendue de ses connaissances dans maints domaines du savoir humain transmuent Césaire en un poète des plus difficiles. En effet, nourri de littératures grecque, latine et française, Césaire use d'un vocabulaire aussi vaste que spécialisé pour exprimer avec des mots européens des paroles africaines. Néanmoins l'usage des mots rares et des néologismes ne saurait étonner dans l'exacte mesure où la poésie renâcle fondamentalement à se servir des expressions qui semblent inhérentes à la prose. Que la poésie césairienne soit difficultueuse, cela est sans conteste, car l'hermétisme auquel appartiendrait son œuvre participe d'une esthétique de rupture avec la prosodie française; ce dont témoigne l'usage délibéré de "l'agrammaticalité,"'18 d'images surréalistes, d'archaïsmes et de néologismes déroutants. A cela s'ajoutent un vocabulaire exotique, une symbolique nourrie des réalités antillaises et une écriture sophistiquée. D'aucuns regardent la poésie de Césaire comme une poésie savante, mettent l'accent sur son abord difficile et invitent le chantre des souffrances nègres à produire des œuvres poétiques qui satisfassent l'entendement de la vox-populi. Cependant le propos de Césaire ne consiste nullement à répondre aux inclinations contradictoires d'un public, dût-il être populaire. Le poète s'explique mal l'éreintement dont il est victime. Par le biais d'un plaidoyer pro domo, il s'inscrit en faux contre le prétendu ésotérisme de sa poésie:

Oui, je sais qu'on me trouve obscur, voire maniéré, soucieux. d'exotisme. C'est absurde. Je suis antillais. Je veux une poésie concrète, très antillaise, martiniquaise. Je dois nommer les choses martiniquaises, les appeler par leur nom. ${ }^{19}$

On le voit, ces lignes ne sauraient prétendre à l'exhaustivité; à tout le moins tâchent-elles de réhabiliter une poésie considérée jusque-là comme inintelligible. Il n'empêche que Césaire "compris ou non, bien ou mal interprété ... exerce une influence considérable sur le développement des lettres françaises." ${ }^{20} \mathrm{Au}$ vrai, il est des obstacles apparemment insurmontables auxquels se heurte l'herméneutique de la poésie césairienne. Toutefois, Césaire, à l'inverse de Mallarmé ne passe pas pour un héraut de l'hermétisme. Bien au contraire, sa poésie prophétique transcende les limites étroites d'un lyrisme individuel, s'affranchit du corset surréaliste et embouche la trompette de la négritude dans l'intention de mourir à la servitude blanche pour renaître à la dignité nègre. Du reste, 
"l'originalité de Césaire est d'avoir coulé son souci étroit et puissant de nègre, d'opprimé et de militant dans le monde de la poésie la plus destructrice, la plus libre et la plus métaphysique."21

Belle comme une bouffée d'oxygène, la poésie de Césaire exprime les souffrances universelles, chante les heurs et les malheurs des Noirs et annonce des lendemains meilleurs. De cette poésie savante et prophétique naît un théâtre qui aspire à l'universel.

Université Gaston Berger Saint-Louis, Sénégal 


\section{Notes}

1. André Breton, préface, Cahier d' un retour au pays natal, d'Aimé Césaire, (Paris: Présence Africaine, 1983) 80.

2. Jean-Claude Bajeux, Antilia retrouvée. Claude McKay Luis Palés, Aimé Césaire, poètes noirs antillais, (Paris: Editions caribéennes, 1983) 190.

3. Aimé Césaire, Cahier d'un retour au pays natal 22.

4. Jean-Michel Devésa, "Un grand poète noir," Europe 832-833 (1998): 5 .

5. Jean-Paul Sartre, "Orphé Noir," Anthologie de la nouvelle poésie nègre et malgache de langue française, éd. Léopold Sédar Senghor, (Paris: PUF, 1969) xxxi.

6. Sartre, Anthologie xxx.

7. Aimé Césaire, Cahier d'un retour au pays natal 48.

8. Aimé Césaire, Poésie, (Paris: Seuil, 1994) 5.

9. Le titre de son premier recueil poétique: Les Armes miraculeuses, (Paris: Gallimard,1946).

10. M. aM. Ngal, Ainé Césaire. Un homme à la recherche d'une patrie, (Dakar-Abidjan: Les nouvelles éditions africaines, 1975) 156.

11. Aimé Césaire, Cahier d'un retour au pays natal 38.

12. Aimé Césaire, Cahier d'un retour au pays natal 46.

13. Sartre, Anthologie xvii.

14. Jean-Claude Bajeux, Antilia retrouvée 290.

15. David Diop, Coups de pilon, (Paris: Présence Africaine, 1973) 72.

16. Aimé Césaire, Et les chiens se taisaient, (Paris: Présence Africaine, 1956-8)91.

17. J.P. Makouta-Mboukou, Introduction à l'étude du roman négroafiricain de langue française. Problèmes culturels et littéraires, (DakarAbidjan-Lomé: Les nouvelles éditions africaines, 1980) 27.

18. Jean-Claude Bajeux, Antilia retrouvée 277.

19. Césaire, cité dans Antilia retrouvée 275.

20. Janheinz Jahn, Muntu, l'homme africain et la culture néoafricaine, (Paris: Seuil, 1961) 126.

21. Jean-Paul Sartre, Situations III. Lendemains de guerre, (Paris: Gallimard, 1949) 260. 


\section{Works Cited}

Bajeux, Jean-Claude. Antilia retrouvée. Claude McKay Luis Palés, Aimé

Césaire, poètes noirs antillais. Paris: Editions caribéennes, 1983.

Césaire, Aimé. Cahier d'un retour au pays natal. Paris: Présence Africaine, 1983.

—. Et les chiens se taisaient. Paris: Présence Africaine, 1956-8.

-.Pósie. Paris: Seuil, 1994.

Devésa, Jean-Michel. "Un grand poète noir à l'assaut du ceil." Europe 832-833(1998): 3-7.

Diop, David. Coups de pilon. Paris: Présence Africaine, 1973.

Jahn, Janheinz. Muntu, l' homme africain et la culture néo-africaine. Paris: Seuil, 1961.

Makouta-Mboukou, J.P. Introduction à l'étude du roman négro-africain de langue française. Problèmes culturels et littéraires. Dakar-AbidjanLomé: Les nouvelles éditions africaines, 1980.

Ngal, M. aM. Aimé Césaire. Un homme à la recherche d'une patrie. Dakar-Abidjan: Les nouvelles éditions africaines, 1975.

Sartre, Jean-Paul. "Orphé Noir." Anthologie de la nouvelle poésie nègre et malgache de langue française. Ed. Léopold Sédar Senghor. Paris: PUF, 1969.

—. Situations III. Lendenains de guerre, Paris: Gallimard, 1949.

Sédar Senghor, Léopold, éd. Anthologie de la nowvelle poésie nègre et malgache de langue française. Paris: PUF, 1969. 